through the whole cloud, but is predominantly located close to the near-face of the cloud. Dust excess determinations show that the Trapezium Cluster stars sampled here contain a typical proportion of classical (dust-excess) T Tauri stars compared with naked (no dust-excess) $\mathrm{T}$ Tauri stars for a young stellar population. Approximately one-third of our sample have insignificant dust excesses.

Calcium II IR triplet emission is observed in members of our Trapezium Cluster sample. We judge that the strengths of the triplet features imply a circumstellar disk origin for the emission. The frequency of calcium triplet emitting stars is estimated for our sample. We compare this estimate with the proportion of triplet emitters in a sample of Chamaeleon pre-main sequence stars. We find that $20-30 \%$ of classical $\mathrm{T}$ Tauri stars in the two populations exhibit triplet emission; the frequency of triplet emission in the Trapezium Cluster sample is found to be comparable with that in Chamaeleon.

We perform an approximate dynamical analysis of the Trapezium Cluster star-forming region using our estimates for stellar mass and age. The low mass cluster is found to be at an early stage in its dynamical evolution, and has not had time to completely relax as a system and lose its initial characteristics. It is too young, therefore, to exhibit mass segregation, and the observed isothermality of the stars is proposed here to arise from the distribution of the clumps from which the stars have formed. The high mass stars considered separately are determined to be old enough to have relaxed as a system. We find that, if the $\Theta^{1}$ Ori stars are located centrally with respect to the low mass cluster, then they are most likely to have formed in their present locations rather than have arrived there from larger radii through dynamical friction processes. In addition, the binding energies of the two high mass binary systems are found to be almost forty times the energy of the low mass cluster. It is suggested that the binary energies must have been acquired through their formation processes instead of through dynamical interations with the low mass cluster. Thus, present characteristics of the stellar population of the Trapezium Cluster directly relate to the conditions under which the cluster formed, and are not due to dynamical processes among the stars themselves. The total stellar mass determined for the low mass cluster is estimated to be sufficient to bind the cluster at this time. This remains true even with future removal of gas from the region. However, continued dynamical intereactions may lead to the eventual dissipation of the low mass cluster.

\section{A STUDY OF SOME NUCLEAR REACTIONS RELEVANT TO OXYGEN BURNING IN SUPERNOVAE}

\section{Alistair F. Scott}

Physics Department, University of Melbourne, Parkville Vic 3052 February 1993

This thesis describes the measurement of absolute cross section and calculations of thermonuclear reactions rates for a number of reactions of importance to explosive and carbon burning.

The results of calculations made with the statistical model HAUSER $* 4$ are compared with the experimental results.

The significance of the calculated thermonuclear reaction rates for stellar nucleosynthesis is discussed. All rates have been parameterized for use in nucleosynthesis network calculations.

\section{NEAR AND FAR INFRARED OBSERVATIONS OF PROTOSTARS AND DARK CLOUDS}

\section{Mark Gerard Suters}

Physics Department, University of Wollongong,

Wollongong NSW 2500

December 1992

Far infrared point source and extended emission data from the Infrared Astronomical Satellite (IRAS) survey are used to investigate the properties of star formation in the regions of high galactic latitude dark cloud complexes. The properties of individual sources are examined using near infrared spectroscopy and broad band spectral energy distributions.

The IRAS signature of star formation is derived by comparing the far infrared colours of a sample of protostars with those of other common far infrared objects. The quality of the IRAS data is ignored for the purposes of this investigation. The criteria developed for identifying protostars from the IRAS Point Source Catalogue discriminates against most non-protostellar objects, with the exception of galaxies and HII regions. Objects identified as protostellar according to other criteria are also likely to be identified by the criteria developed.

Extended emission data in the far infrared is used to estimate the column density and temperature of several dark cloud complexes, and the optical extinction in the same regions is estimated with the Guide Star Catalogue. Temperature and column density share an inverse relationship: cloud cores are characterised by column densities above $10^{24}$ hydrogen atoms $\mathrm{m}^{-2}$ and temperatures around $20 \mathrm{~K}$, while the inter cloud medium has column densities below $10^{23}$ atoms $\mathrm{m}^{-2}$ and temperatures above $50 \mathrm{~K}$. The column density, as measured by IRAS, and the optical extinction appear to be related up to values of around $10^{25}$ atoms $\mathrm{m}^{-2}$ and 5 magnitudes respectively, but the IRAS detectors appear insensitive to material at higher densities than these.

Near infrared spectra of a variety of objects chosen for their youth, including IRAS sources which satisfy the protostar criteria, are investigated. These spectra are categorised into three distinct groups of increasing youth:

1. T Tauri-like spectra, with flat $\mathrm{H}$ and $\mathrm{K}$ band continua, lacking both $\mathrm{Br}-\gamma$ emission and $\mathrm{CO}$ absorption;

2. FU Ori-like spectra, with $\mathrm{CO}$ absorption, $1.9 \mu \mathrm{m}$ water absorption, and possibly $\mathrm{Br}-\gamma$ emission, and

3. embedded protostar spectra, with red continuum, and perhaps $\mathrm{Br}-\gamma$ emission, but lacking $\mathrm{CO}$ absorption.

The spectral energy distributions (SEDs) of the sources are also investigated, using data at wavelengths from 0.3 $\mu \mathrm{m}$. All of the objects studied in the near infrared have SEDs characteristic of either T Tauri stars or of embedded protostars. There seems little correlation between the evolutionary status assigned to an object on the basis of its SED and that assigned from near infrared spectral features, perhaps because the shape of the SED is independent of the evolution of the central object, and depends on geometric considerations such as the orientation of the circumstellar disk in relation to the line of sight from the Earth. 